\title{
Current and future aspects of TIM-3 as biomarker or as potential targeted in non-small cell lung cancer scope: is there a role in clinical practice?
}

\author{
Ramon Andrade De Mello ${ }^{1,2,3} \wedge$, Jin-Hui Zhu ${ }^{4}$, Jairo Iavelberg ${ }^{1}$, Artur Henrique Potim ${ }^{1}$, Débora Simonetti ${ }^{1}$, \\ José Antônio Silva Jr${ }^{1}$, Pedro Castelo-Branco ${ }^{3}$, Daniel Humberto Pozza ${ }^{5}$, Carla Chizuru Tajima ${ }^{6}$, Maria \\ Tolia $^{7}$, Georgio Antoniou ${ }^{8}$ \\ ${ }^{1}$ Post-graduation Program in Medicine, Precision Oncology \& Health Economics Research Group (OncoPRECH), Faculty of Medicine, Nine of \\ July University (UNINOVE), São Paulo, Brazil; ${ }^{2}$ Division of Medical Oncology/Oncotorax Group, Escola Paulista de Medicina, Federal University \\ of São Paulo (UNIFESP), São Paulo, Brazil; ${ }^{3}$ Algarve Biomedical Centre, Department of Biomedical Sciences and Medicine, University of \\ Algarve (UALG), Faro, Portugal; ${ }^{4}$ Department of Surgery, Second Affiliated Hospital, Zhejiang University School of Medicine, Hangzhou, China; \\ ${ }^{5}$ Department of Biomedicine \& I3S, Faculty of Medicine, University of Porto (FMUP), Porto, Portugal; ${ }^{6}$ Hospital São José \& Hospital São Joaquim- \\ Beneficência Portuguesa de São Paulo, São Paulo, Brazil; ${ }^{7}$ Department of Radiotherapy/Radiation Oncology, Faculty of Medicine, School of Health \\ Sciences, University of Thessaly, University Hospital of Larisa, Biopolis, Greece; ${ }^{8}$ Division of Thoracic Oncology, Mont Vernon Cancer Center, \\ London, UK \\ Correspondence to: Ramon Andrade De Mello, MD, PhD, FACP. Algarve Biomedical Centre, University of Algarve, Campus de Gambelas, edifício 2, \\ ala norte, 8005-139, Faro, Portugal. Email: ramondemello@gmail.com. \\ Comment on: Jia K, He Y, Dziadziuszko R, et al. T cell immunoglobulin and mucin-domain containing-3 in non-small cell lung cancer. Transl Lung \\ Cancer Res 2019;8:895-906.
}

Submitted May 01, 2020. Accepted for publication Jun 15, 2020.

doi: $10.21037 /$ tlcr-20-625

View this article at: http://dx.doi.org/10.21037/tlcr-20-625

Lung cancer is an aggressive disease with a high rate of mortality (1). Non-small cell lung cancer (NSCLC) constitutes approximately $85 \%$ of all histology types (2). In the 1990's, chemotherapy was the standard of care for the treatment of metastatic NSCLC (3). Since 2005, targeted therapies have emerged as a new cornerstone in the treatment of NSCLC. These include epidermal growth factor receptor tyrosine kinase inhibitors (EGFRTKI) such as gefitinib, erlotinib, afatinib, osimertinib, and dacomitinib (4) as well as the anaplastic lymphoma kinase (ALK) inhibitors crizotinib, alectinib, ceritinib, brigatinib and lorlatinib $(5,6)$. Improving our understanding of tumor biology has therefore become a fundamental issue among oncologists to optimize these novel treatment strategies (7). In 2018, James P. Allison and Tasuku Honjo (8) won the Nobel prize for their research on the mechanisms of the tumor immune escape, which led to the first immunotherapy drugs to be utilized in clinical practice: nivolumab and ipilimumab $(3,9)$. The Karolinska Institute Nobel Prize Committee declared that Allison and Honjo's findings constituted the fourth cornerstone of cancer treatment, alongside surgery, radiotherapy and chemotherapy $(1,9)$.

Interestingly, ipilimumab, an anti-CTLA-4 monoclonal antibody, was the first drug targeting an immune checkpoint on $\mathrm{T}$ cells that achieved important responses in patients with advanced melanoma. It was therefore approved by the Food and Drug Administration (FDA) in 2011 for the treatment of metastatic melanoma (10). In 2018, Hellmann et al. (11) described the role of ipilimumab plus nivolumab for the treatment of lung cancer with a high tumor mutation burden (11). First-line nivolumab plus ipilimumab achieved significantly longer progression-free survival (PFS) than standard chemotherapy in advanced

^ ORCID: 0000-0002-9640-4573. 
NSCLC patients harboring a high tumor mutation burden, irrespective of PD-L1 expression (11). Additional drugs such as pembrolizumab $(12,13)$, atezolizumab $(14,15)$ and durvalumab (3) were subsequently approved alone or in combination with chemo/targeted therapy for the treatment of locally-advanced and advanced NSCLC.

Recently, improved understanding of the molecular biology of NSCLC has produced promising new biomarkers (16). Among of that, the understanding of a potential biomarker which could improve the inhibition of the immune check points raised as a new challenge in the clinical practice. Apart of PD-L1 expression, other molecules have been study with potential role in this field. One interesting example is TIM-3. It is a type I transmembrane protein that it is related to a novel cell surface molecules that would identify IFN- $\gamma$-producing Th 1 and Tc1 cells (17). One of TIM3 main role is encode the proteins TIM-1, TIM-3 and TIM-4 (18). It is related to both autoimmune and allergic diseases $(17,18)$ and it is expressed on IFN- $\gamma$-producing $\mathrm{T}$ cells and FoxP $3+$ Treg cells, including macrophages and dendritic cells, where it has been shown to suppress their responses upon interaction with their ligand (17). More recently, TIM-3 emerged as a potential role in immune check points inhibitors, since It has been shown that it could enhances anti-tumor immunity and suppresses tumor growth in several preclinical tumor models (17). Several anti-TIM-3 targeted drugs are currently in clinical trials and they are considered promising for enhancing anti-PD-1/PDL-1 treatment when used in combination framework $(17,18)$.

In 2019, Jia et al. published a study in this prestigious scientific journal (18) that sought to elucidate the association of TIM-3 expression patterns and NSCLC immunotherapy, and how these correlated with overall survival (OS) (18). The authors assessed 139 surgically resected NSCLC specimens from a Polish University Oncology Center (18). The histological slides were independently reviewed by two senior pathologists (18). TILs were confirmed as TIM-3 positive only when staining was greater than $10 \%$ (18). The fraction of TILs was divided into three levels: $<30 \%$ (low), 30-60\% (medium) and $>60 \%$ (high). In tumor cells, the cutoff was set lower $(>5 \%)$ due to the rare presence of TIM-3. The number of lymphocytes within each histology spot was also determined. Out of all samples tested, 9 (6.5\%) had positive TIM-3 expression on tumor cells $(>5 \%$ staining) and $11(7.9 \%)$ had TIM-3 positive TILs (>10\% staining) (18). Interestingly, TIM-3 expression on TILs was also positively correlated with the expression of PD-L1 on tumor cells as well as the expression of TIM-3 on tumor cells and the percentage of TILs (18). Despite of that, the expression of TIM-3 on tumor cells only correlated with the expression of PD-L1 (18).

The expression level of TIM-3 on tumor cells failed to predict relapsed free survival (RFS) or OS, although the TIM-3 positive patients demonstrated a non-significant reduction in survival time (18). in stage I-II patients, the findings did show that TIM-3 on TILs had a significant prognostic value. The Cox regression analysis showed no association of TIM-3 expression with TILs or TCs. Cancer stage was the only identified variable that was related to both RFS and OS. In addition, TIL expression of TIM-3 showed significant prognostic value for OS (18). This study suggested that TIM-3 plays a key role in the dysfunction of $\mathrm{T}$ cell immunity and could be related to tumor aggressiveness. The findings of this study suggest that further investigation of the upstream signal pathways that induce the co-expression of TIM-3 and PD-1/L1 are warranted (18).

Currently, the synergistic effects in restoring antitumor immunity in preclinical animal models could give a biological rational for the combination therapies based on blockade of TIM-3 and other inhibitory molecules, such as PD-1 (17). In some pre-clinical and clinical setting, multiple chronic viral infections, including HIV, HCV, HBV, Friend virus, and LCMV suggested a connection between elevated TIM-3 expression and exhausted CD8+ T cells. There are some evidence that TIM-3 acts as a checkpoint inhibitor for anti-tumor immunity, since its showed an association with $\mathrm{T}$ cell exhaustion in malignant tumor environment (17). The inhibition of TIM-3 by monoclonal anti-bodies (mAbs) is being assessed in different strategies of drugs combinations, which might include immunochemotherapy and/or different immune-checkpoint inhibitors associations (17). Imuno-chemotherapy is one of the most promising approaches since the hypothesis that cytotoxic chemotherapy may have synergic effects with the tumor immunogenicity (16). Paz-Ares and colleagues published in 2018 the keynote 407 clinical trial that assessed 559 previous untreated metastatic squamous NSCLC for the randomization of pembrolizumab plus carboplatin and either nanoparticle albumin-bound [nab]-paclitaxel or paclitaxel for four cycles followed by pembrolizumab maintenance. After 7.8 months follow up, the median OS as 15.9 months to pembrolizumab-chemotherapy group versus 11.3 months to placebo-chemotherapy combination group. Personally, our clinical experience is very positive with this 
pembrolizumab-carboplatin-nab-paclitaxel regimen with also complete response patients and manageable toxicity profile. Either in 2018, Leena Gandhi was the first author of an interesting clinical trial named KEYNOTE 189 which assessed 616 metastatic non-squamous NSCLC without harboring sensitizing EGFR mutation or ALK fusions eligible for the first line systemic treatment (16). Patients were randomized to receive pembrolizumab versus placebo combined with pemetrexed-platinum regimen for four cycles, followed by pembrolizumab versus placebo plus pemetrexed maintenance. After 10.5 months follow up, there was a significant improvement in OS of $51 \%$ (HR $0.49, \mathrm{P}<0.001)$ for the pembrolizumab combination group. Despite the reported grade 3 or higher adverse events of $67.7 \%$ in combination group versus $65.8 \%$ in placebo group, our patients experienced a very good tolerable profile with KEYNOTE 189 protocol since we have a multi-disciplinary specializing supportive oncology doctors' team to see patients weekly in outpatient clinics, such as dermatologist, palliative specializing doctor, geriatrics, endocrinologist and cardiologist. However, the search for putative predictive and prognostic biomarkers is still need to personalize treatment for the right patient in order to obtain improved clinical outcomes.

In 2018, Kato et al. published a study that assessed nivolumab in advanced esophageal-gastric cancer setting (19) which evaluated biomarkers throughout peripheral blood collected from 20 patients at a single institution (19). There was no significant differences in general examinations or tumor markers at baseline (19). T-cell subsets were analyzed by flow cytometry using stored peripheral blood mononuclear cells (PBMCs) (19). Authors assessed the expression of the immune checkpoints PD-1, TIM-3, OX40, 4-1BB, LAG3, and ICOS, and the activation markers Ki-67 and CD103 on CD4 and CD8 T cells and clinicopathological factors. After the first cycle, levels of TIM-3 were significantly higher in CD4 and CD8 $\mathrm{T}$ cells responding patients than non-responding patients (19). In this study, changes in TIM-3+CD4 levels had a significant impact on OS rates (19). Therefore, TIM-3 expression could be considered as a potential predictive and prognostic biomarker in this patient population. In the framework of advanced NSCLC, systemic treatments have undergone rapid change. The combination of immune-chemotherapies and targeted therapies have attained an important role in clinical practice. The use of broad Next Generation Sequence biomarker panels presents a promising strategy for personalizing medicine. TIM-3 appears to be a potential new biomarker that will further enable the individual tailoring of immunetherapy protocols and thus improve patient outcomes (17) as well as increasing cost-effectiveness in the advanced NSCLC setting $(15,17)$. However, further large phase I-III clinical trials are warranted to validate this hypothesis.

\section{Acknowledgments}

We would like to acknowledge the post-graduation program in medicine of the Nine of July University (UNINOVE), São Paulo, Brazil, for their technical support.

Funding: We would like to acknowledge CNPQ research grant 402621/2016-6.

\section{Footnote}

Provenance and Peer Review: This article was commissioned by the editorial office, Translational Lung Cancer Research. The article did not undergo external peer review.

Conflicts of Interest: All authors have completed the ICMJE uniform disclosure form (available at http://dx.doi. org/10.21037/tlcr-20-625). RADM serves as an unpaid editorial board member of Translational Lung Cancer Research from Jan 2020 to Dec 2021. RADM reports Consultant/ advisory board for Pfizer, Zodiac, MSD, European School of Oncology (ESO); Speaker Honoraria from Astrazenenca, Novartis, Merck, Astellas, ESO; volunteer speaker role for ASCO 2016-2019; Faculty for the Educational Committee of the European Society for Medical Oncology (ESMO), 2018-2022; Faculty for the Cancer Educational Committee of the American Society of Clinical Oncology (ASCO), 2016-2019; Educational Grants: Merck-Group; Travel Grant: Astellas. Research Grant: Conselho Nacional de Desenvolvimento Científico e Tecnológico (CNPQ), Brazil, number 402621/2016-6. Expert honoraria from National Science Center, Poland, and National Medical Research Council, Singapore. Honorary Grant Reviewer for British Lung Foundation, United Kingdom. The other authors have no conflicts of interest to declare.

Ethical Statement: The authors are accountable for all aspects of the work in ensuring that questions related to the accuracy or integrity of any part of the work are appropriately investigated and resolved.

Open Access Statement: This is an Open Access article distributed in accordance with the Creative Commons 
Attribution-NonCommercial-NoDerivs 4.0 International License (CC BY-NC-ND 4.0), which permits the noncommercial replication and distribution of the article with the strict proviso that no changes or edits are made and the original work is properly cited (including links to both the formal publication through the relevant DOI and the license). See: https://creativecommons.org/licenses/by-nc-nd/4.0/.

\section{References}

1. Gouvinhas C, De Mello RA, Oliveira D, et al. Lung cancer: a brief review of epidemiology and screening. Future Oncol 2018;14:567-75.

2. Bryce AH, Rao R, Sarkaria J, et al. Phase I study of temsirolimus in combination with EKB-569 in patients with advanced solid tumors. Invest New Drugs 2012;30:1934-41.

3. de Mello RA, Veloso AF, Catarina PE, et al. Potential role of immunotherapy in advanced non-small-cell lung cancer. Onco Targets Ther 2016;10:21-30.

4. De Mello RA, Escriu C, Castelo-Branco P, et al. Comparative outcome assessment of epidermal growth factor receptor tyrosine kinase inhibitors for the treatment of advanced non-small-cell lung cancer: a network metaanalysis. Oncotarget 2017;9:11805-15.

5. Aguiar Jr P, Del Giglio A, Perry LA, et al. Costeffectiveness and budget impact of lung cancer immunotherapy in South America: strategies to improve access. Immunotherapy 2018;10:887-97.

6. Ando K, Akimoto K, Sato H, et al. Brigatinib and Alectinib for ALK Rearrangement-Positive Advanced Non-Small Cell Lung Cancer With or Without Central Nervous System Metastasis: A Systematic Review and Network Meta-Analysis. Cancers 2020;12:942.

7. Wang $\mathrm{Y}, \mathrm{Zou} \mathrm{S}, \mathrm{Zhao} Z$, et al. New insights into small cell lung cancer development and therapy. Cell Biol Int 2020. doi: 10.1002/cbin.11359.

8. Chamoto K, Al-Habsi M, Honjo T. Role of PD-1 in immunity and diseases. Curr Top Microbiol Immunol 2017;410:75-97.

9. Wei SC, Duffy CR, Allison JP. Fundamental Mechanisms of Immune Checkpoint Blockade Therapy. Cancer Discov 2018;8:1069-86.

10. Hodi FS, O'Day SJ, McDermott DF, et al. Improved survival with ipilimumab in patients with metastatic melanoma. N Engl J Med 2010;363:711-23.

11. Hellmann MD, Ciuleanu T-E, Pluzanski A, et al. Nivolumab plus ipilimumab in lung cancer with a high tumor mutational burden. $\mathrm{N}$ Engl J Med 2018;378:2093-104.

12. Paz-Ares L, Luft A, Vicente D, et al. Pembrolizumab plus chemotherapy for squamous non-small-cell lung cancer. $\mathrm{N}$ Engl J Med 2018;379:2040-51.

13. Mok TS, Wu YL, Kudaba I, et al. Pembrolizumab versus chemotherapy for previously untreated, PD-L1-expressing, locally advanced or metastatic non-small-cell lung cancer (KEYNOTE-042): a randomised, open-label, controlled, phase 3 trial. Lancet 2019;393:1819-30.

14. Reck M, Mok TS, Nishio M, et al. Atezolizumab plus bevacizumab and chemotherapy in non-small-cell lung cancer (IMpower 150): key subgroup analyses of patients with EGFR mutations or baseline liver metastases in a randomised, open-label phase 3 trial. Lancet Respir Med 2019;7:387-401.

15. Zhou K, Zhou J, Huang J, et al. Cost-effectiveness analysis of atezolizumab plus chemotherapy in the first-line treatment of extensive-stage small-cell lung cancer. Lung cancer 2019;130:1-4.

16. Puhr HC, Ilhan-Mutlu A. New emerging targets in cancer immunotherapy: the role of LAG3. ESMO open 2019;4:e00482.

17. Friedlaender A, Addeo A, Banna G. New emerging targets in cancer immunotherapy: the role of TIM3. ESMO open 2019;4:e000497.

18. Jia K, He Y, Dziadziuszko R, et al. T cell immunoglobulin and mucin-domain containing-3 in non-small cell lung cancer. Transl Lung Cancer Res 2019;8:895.

19. Kato R, Yamasaki M, Urakawa S, et al. Increased Tim$3+\mathrm{T}$ cells in PBMCs during nivolumab therapy correlate with responses and prognosis of advanced esophageal squamous cell carcinoma patients. Cancer Immunology, Immunotherapy 2018;67:1673-83.

Cite this article as: De Mello RA, Zhu JH, Iavelberg J, Potim AH, Simonetti D, Silva JA Jr, Castelo-Branco P, Pozza DH, Tajima CC, Tolia M, Antoniou G. Current and future aspects of TIM-3 as biomarker or as potential targeted in non-small cell lung cancer scope: is there a role in clinical practice? Transl Lung Cancer Res 2020;9(6):2311-2314. doi: 10.21037/tlcr-20-625 